\title{
Extended Spectrum Beta Lactamase and Metallo Beta Lactamase Producing Pseudomonas aeruginosa at Tertiary Care Hospital of Nepal
}

\author{
Pallavi Shrestha ${ }^{1}$, Saroj Sharma ${ }^{2}$, Roshani Maharjan ${ }^{3 *}$ \\ ${ }^{1}$ Department of Microbiology, St. Xavier's College, Maitighar, Kathmandu, Nepal \\ ${ }^{2}$ Kanti Children's Hospital, Maharjgunj, Kathmandu, Nepal \\ ${ }^{3}$ Department of Microbiology, Tri-Chandra Multiple College, Ghantaghar, Kathmandu, Nepal
}

*Corresponding author: Roshani Maharjan, Department of Microbiology, Tri-Chandra Multiple College,

Ghantaghar, Kathmandu, Nepal. E-mail: roshani_mh@hotmail.com

\begin{abstract}
Objective: To assess the prevalence of extended spectrum beta lactamase (ESBL) and metallo beta lactamase (MBL) producing Pseudomonas aeruginosa from pus samples.

Methods: A cross-sectional study was conducted at Kanti Children's Hospital, Kathmandu, Nepal during which 316 pus samples were collected and tested using standard microbiological procedures. Antibiotic Susceptibility Test (AST) was done by Kirby-Bauer disk diffusion method and the detection of ESBL and MBL production were done using Ceftazidime/clavulanic acid combined disk test and ImipenemEthylenenediaminetetraacetic acid combined disk test respectively as per CLSI guideline 2014.

Results: The prevalence rate of $P$. aeruginosa was found to be $7.9 \%$ in pus samples. Out of $25 P$. aeruginosa isolates $9(36 \%)$ were ESBL producers and 2(8\%) were MBL producers. ESBL producers were predominant in the age group 2-3 years (33.3\%) and in male patient (55.6\%).Out of $2 \mathrm{MBL}$ producing $P$. aeruginosa, 1(50\%)was isolated from the age group below 2 years and male patient and $1(50 \%)$ from the age group $8-9$ years and female patient. $96 \%$ of isolates showed sensitive to Polymyxin B.

Conclusion: The study showed increasing trend of ESBL and MBL production in P. aeruginosa so constant survey of prevalence of ESBL and MBL producing isolates is essential to control and manage spread of these isolates in different units of health institutions.
\end{abstract}

Key words: Pus, antibiotic susceptibility test, ESBL, MBL, Pseudomonas aeruginosa.

\section{INTRODUCTION}

Multidrug-resistant Pseudomonas aeruginosa, a major pathogen in pyogenic infections (Soumya and Nagmoti 2017) are serious problems to the successful treatment of the wounds leading to complications sometime even fatal sepsis.

P. aeruginosa is intrinsically resistant to most of the drugs making the therapeutic choices limited for its treatment (Murray et al. 2015). The acquisition of ESBL and MBL producing genes by $P$. aeruginosa has made the bacteria resistant to the antibiotics among the limited choice. Thus, making the treatment of infections caused by $P$. aeruginosa difficult or impossible to treat and increasing global threat in community and hospital settings (Ansari

Date of Submission: August 3, 2018

Published Online: January 2019 et al. 2016; Hirsh and Tam 2010; Solomon et al. 2017).

ESBLs are plasmid-mediated beta-lactamase that confer resistance to the penicillins, first-, second-, and third-generation cephalosporins, and Aztreonam and are inhibited by $\beta$-lactamase inhibitors such as clavulanic acid (Poudyal et al. 2011). The exposure of bacterial strains to a multitude of beta-lactams has induced mutation of beta-lactamase in many bacteria, expanding their activity even against carbapenems by the production of MBL carbapenamases which require zinc divalent cation, as cofactor for enzyme activity and are able to hydrolyze all $\beta$ - lactams except monobactam and known to be inhibited by chelating divalent cations like Ethylenediaminetetraacetic acid (EDTA).

Date of Acceptance: November 15, 2018

DOI: https:/ / doi.org/10.3126/tujm.v5i0.22301 
The ESBL and MBL producing P. aeruginosa infections have high mortality rates and have emerged worldwide rapidly. A very few study on ESBL and MBL producing $P$. aeruginosa has been conducted in Nepal thus necessitating the extensive study on its prevalence. This study was conducted with an objective toassess the prevalence of ESBL and MBL producing P. aeruginosa from pus samples.. This study would help to plan a proper hospital infection control strategy to prevent the spread of these isolates.

\section{MATERIALS AND METHODS}

A hospital based descriptive cross sectional study was carried out at Kanti Children's Hospital, Maharjgunj, Kathmandu Nepal from August 2015 to January 2016. This study included patients of age group below 14 years of both sex. A total of 316 samples sent for routine investigation were processed. The study protocol was approved by the institutional review committee (Ref no: 117-072/73) of Kanti Children's Hospital, Kathmandu.

Pus sample in cotton swab and aspirated pus form was inoculated on to MacConkey Agar (MA), Blood Agar (BA) and Cetrimide agar (Himedia) plates according to standard microbiological procedure (Forbes et al. 2007). Identification of $P$. aeruginosa was done by using conventional biochemical tests. Antimicrobial sensitivity testing was performed on Mueller-Hinton agar plates by Kirby-Bauer disk diffusion method as described by Clinical Laboratory Standards Institute guidelines (CLSI 2014).

\section{ESBL detection}

The isolates were screened for possible ESBL production using Ceftazidime $(30 \mu \mathrm{g})$ as per CLSI 2014 guidelines. According to the guidelines, isolates showing cefpodoxime $<17 \mathrm{~mm}$, ceftazidime $<22 \mathrm{~mm}$, aztreonam $\leq 27 \mathrm{~mm}$, cefotaxime $<27 \mathrm{~mm}$, and ceftriaxone $<25 \mathrm{~mm}$ are the possible ESBL producing strains. ESBL production was confirmed among the suspected bacterial strain using combined disk (CD) assay, an increase in zone

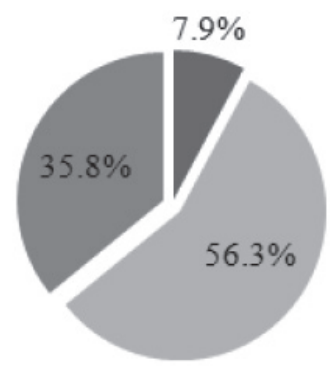

Figure 1: Distribution of Pseudomonas aeruginosa in pus samples size of $\geq 5 \mathrm{~mm}$ from either of the combination disk i.e. clavulanate containing disk indicated the presence of ESBL in the test organisms.

\section{MBL detection}

For the detection of production of MBL, combined disk test (CDT) was performed using two Imipenem disks $(10 \mu \mathrm{g})$, one containing $10 \mu \mathrm{l}$ of $0.1 \mathrm{M}(292 \mu \mathrm{g})$ anhydrous EDTA (Hi-Media, India). Disks were placed $25 \mathrm{~mm}$ apart and an increase in zone diameter of $>4$ $\mathrm{mm}$ around the IPM-EDTA disk compared to that of the IPM disk alone was considered positive for MBL production. These isolates were considered to be of the MBL positive phenotype (Lee et al. 2003).

\section{RESULTS}

Among 316 non repetitive pus samples processed, $P$. aeruginosa was isolated from 25(7.9\%) samples (Figure 1). Maximum number of isolates were obtained from females $13(52 \%)$ and in the age group 4-5 years $8(32 \%)$. The association between culture positivity of $P$. aeruginosa isolates with age and gender was found statistically insignificant $(\mathrm{p}>0.05)$. Highest percentage of Pseudomonas aeruginosa isolates were sensitive to Polymyxin B (96\%), followed by Imipenem (92\%), Amikacin (88\%) (Table 1).

Among 25 P. aeruginosa isolates, 9(36\%) were ESBL producers and $2(8 \%)$ were MBL producers. Of 21 screening positive $P$. aeruginosa, $9(42.9 \%)$ were confirmed to be ESBL producers by phenotypic methods. There was no association between screening and confirmatory tests ( $p>0.05$ ) (Table 2). ESBL producers were predominant in the age group $2-3$ years $(33.3 \%)$ (Table 3 ) and in male patient $(55.6 \%)$ (Table 4$)$. Out of $2 \mathrm{MBL}$ producing $P$. aeruginosa $1(50 \%$ ) was isolated from the age group below 2 years and male patient and $1(50 \%)$ from the age group 8-9 years and female patient. ESBL production and MBL production was found to be insignificantly associated with age and gender $(\mathrm{p}>0.05)$.

- Pseudomonas aeruginosa

Other Isolates

Culture negative 
Table 1: Antibiotic susceptibility pattern of $P$. aeruginosa

\begin{tabular}{lccc}
\hline \multirow{2}{*}{ Antibiotics } & \multicolumn{3}{c}{ Susceptibility pattern } \\
\cline { 2 - 4 } & Sensitive, N (\%) & Intermediate, N (\%) & Resistant, N (\%) \\
\hline Amikacin & $22(88)$ & 0 & $3(12)$ \\
Piperacillin & $2(8)$ & $6(24)$ & $17(68)$ \\
Piperacillin/Tazobactam & $8(32)$ & $4(16)$ & $13(52)$ \\
Ciprofloxacin & $16(64)$ & $1(4)$ & $8(32)$ \\
Ceftazidime & $10(40)$ & $4(16)$ & $11(44)$ \\
Cefotaxime & $5(20)$ & $10(40)$ & $10(40)$ \\
Imipenem & $23(92)$ & 0 & $2(8)$ \\
Polymyxin B & $24(96)$ & 0 & $1(4)$ \\
\hline
\end{tabular}

Table 2: Screening and confirmation of ESBL production

\begin{tabular}{|c|c|c|c|c|c|c|}
\hline \multirow{2}{*}{ Screening } & \multicolumn{4}{|c|}{ Production of ESBL } & \multirow{2}{*}{ Total } & \multirow{2}{*}{ P-value } \\
\hline & Yes & $\%$ & No & $\%$ & & \\
\hline Positive & 9 & 42.9 & 12 & 57.1 & 21 & \multirow{2}{*}{0.102} \\
\hline Negative & 0 & 0 & 4 & 100 & 4 & \\
\hline Total & 9 & 36 & 16 & 64 & 25 & \\
\hline
\end{tabular}

Table 3: Distribution of ESBL producing $P$. aeruginosa isolates according to patient age

\begin{tabular}{|c|c|c|c|c|}
\hline \multirow{2}{*}{ Age group (Years) } & \multicolumn{2}{|c|}{ ESBL Positive } & \multirow{2}{*}{ Total } & \multirow{2}{*}{$\mathrm{p}$-value } \\
\hline & No & $\%$ & & \\
\hline$<2$ & 2 & 22.2 & 7 & \multirow{8}{*}{0.7} \\
\hline $2-3$ & 3 & 33.3 & 5 & \\
\hline $4-5$ & 2 & 22.2 & 8 & \\
\hline$-6-7$ & 0 & 0 & 0 & \\
\hline$-8-9$ & 1 & 11.1 & 2 & \\
\hline $10-11$ & 0 & 0 & 1 & \\
\hline $13-14$ & 1 & 11.1 & 2 & \\
\hline Total & 9 & 100 & 25 & \\
\hline
\end{tabular}

Table 4: Distribution of ESBL producing isolates according to sex of patient

\begin{tabular}{|c|c|c|c|c|}
\hline \multirow{2}{*}{ Sex } & \multicolumn{2}{|c|}{ ESBL positive } & \multirow{2}{*}{ Total } & \multirow{2}{*}{$\mathrm{p}$-value } \\
\hline & No & $\%$ & & \\
\hline Male & 5 & 55.6 & 12 & \\
\hline Female & 4 & 44.4 & 13 & 0.57 \\
\hline Total & 9 & 100 & 25 & \\
\hline
\end{tabular}

\section{DISCUSSION}

Of the total 316 pus samples processed, P. aeruginosa was isolated from 25 samples. The prevalence rate of $P$. aeruginosa was found to be $7.9 \%$ which is in accordance with the study conducted by Mantravadi et al. 2015 and Upadhyay et al. 2010 that showed $7.5 \%$ and $7.4 \%$ P. aeruginosa growth respectively. Similar study conducted by Dash et al. 2014, Sharma et al. 2016 and
Yakha et al. 2014 showed the prevalence of P. aeruginosa in pus as $13.0 \%, 22.4 \%$ and $12.1 \%$ respectively. This variation may have occurred due to type and number of pus specimen used, source of pus, use of antibiotic, climate and topographical situation of area under study, sanitation and the duration of study.

Maximum number of isolates were obtained from 
females $13(52 \%)$ which is similar to the study carried out by Hassuna et al. 2015. This might be due to large number of females admitted to the hospital than the males. But most of the other similar studies showed greater isolation in males for example Al-Marzoqi and Al-Taee 2013, Biradar et al. 2016 and Sonth et al. 2015. Maximum number of isolates were obtained from the age group $4-5$ years $8(32 \%)$. The study carried out by Hassuna et al. 2015 showed the higher isolation of $P$. aeruginosa from the age group below 10 years. The association between culture positivity of $P$. aeruginosa isolates with age and gender was found statistically insignificant $(\mathrm{p}>0.05)$.

Of 25 isolates 21 (84\%) were screening positive and of 21 screening positive isolates $9(42.8 \%)$ were confirmed to be ESBL producers which is in harmony with the study carried out by Bharti and Sharma 2014.In contrary to our study, Tsering et al. 2009 showed $32.6 \%$ screening positive for ESBL production and all the isolates were positive for ESBL production by phenotypic confirmatory tests.

The issue of ESBL and MBL production is increasing at different rates throughout the world that has become problematic in therapeutic treatment. This study showed the high prevalence of ESBL producing Pseudomonas aeruginosa (36\%) which is in accordance with the study carried out by Ansari et al. 2016 that showed 33.1\% of isolates to be ESBL producer. Similar study carried out in Nepal by Pathak and Pokharel 2015 showed $18.1 \%$ of isolates to be ESBL producer which was low as compared to this study. Similarly, the study of Poudyal et al. 2011 recorded zero percent of $P$. aeruginosa as ESBL producers. This indicated the increasing trend of ESBL production in P. aeruginosa in Nepal.

This study showed the prevalence of MBL producing $P$. aeruginosa to be $8 \%$ which is high as compared to the study conducted in Nepal by Mishra et al. 2012, Shrestha et al. 2011 which showed 3.3\% and 2.4\% of $P$. aeruginosa isolates as MBL producers respectively. Similarly, the study carried out by Ansari et al. 2016 and Khanal et al. 2013 showed $30.9 \%$ and $18.2 \%$ of $P$. aeruginosa isolates as MBL producers respectively. This study documents low prevalence of MBL producing $P$. aeruginosa. But increased use of carbapenems to treat ESBL isolates and horizontal transfer of MBL genes might lead to high prevalence of MBL in future that poses serious therapeutic challenges.

In this study, the highest percentage of ESBL isolates was isolated from the male patient (55.5\%) which is in accordance with a study carried out by Anjum and Mir 2010 where the highest percentage of ESBL isolates was isolated from female. The highest percentage of ESBL isolates was isolated from the age group 2-3 years (33.3\%). ESBL production was found to be insignificantly associated with age and gender $(p>0.05)$.

Out of 2 MBL producing P. aeruginosa 1(50\%) was isolated from the age group below 2 years and male patient and $1(50 \%)$ from the age group 8-9 years and female patient. MBL production was found to be insignificantly associated with age and gender $(\mathrm{p}>0.05)$.

Polymyxin B was found to be the most effective drug against $P$. aeruginosa with $96 \%$ susceptibility which is in accordance with the similar study carried out by Kumar et al. 2014, Sharma et al. 2016 and Tankhiwale 2016 that showed 94\%, 95\% and 94.5\% susceptibility respectively. In a similar study carried out by Bhandari et al. 2012, Parajuli et al. 2014 and Patel and Garala 2014, 63.6\%, 64.2\% and 73\% of P. aeruginosa isolates was sensitive toward Polymyxin B respectively which is less than our findings. In this study, Imipenem (92\%), Amikacin (88\%) and Ciprofloxacin (64\%) were also found to be effective against Pseudomonal infections. Since Ciprofloxacin has fewer side effects and is cheaper than other drugs, it can be recommended as the drug of choice for Pseudomonal infections.

\section{CONCLUSION}

This study showed the increased prevalence of ESBL and MBL production in $P$. aeruginosa which warrants early detection in routine laboratory, immediate infection control, and antibiotic stewardship programs in order to limit the spread of ESBL and MBL positive isolates. The appearance of ESBL and MBL genes and their spread among bacterial pathogens are matters of major concern with regard to the future antimicrobial chemotherapy. Polymyxin B was found to be the most effective drug against $P$. aeruginasa.

\section{ACKNOWLEDGEMENTS}

We are thankful to all the staff of Microbiology Department of Kanti Children's Hospital for their generous help and support. 


\section{CONFLICT OF INTEREST}

The authors declare no conflict of interest.

\section{REFERENCES}

Al-Marzoqi AH and Al-Taee ZM (2013). Pseudomonas aeruginosa: Antibiotic resistance pattern to different isolates in Al-Hillah city, Iraq. J Nat Sci Res 3(3): 69-74.

Anjum F and Mir A (2010). Susceptibility pattern of Pseudomonas aeruginosa against various antibiotics. Afr J Microbiol Res 4(10): 1005-1012.

Ansari S, Dhital R, Shrestha S, Thapa S, Puri R, Chaudhary N, Khatiwada S, and Gautam R (2016). Growing Menace of Antibacterial Resistance in Clinical Isolates of Pseudomonas aeruginosa in Nepal: An Insight of Beta-Lactamase Production. BioMed Res Int: 6437208: 8.

Bhandari S, Banjara MR, Lekhak B ,Bhatta DR and Regmi SR (2012). Multi-Drug and Pan-Drug Resistant Pseudomonas aeruginosa: A Challenge in Post- Antibiotic Era. Nepal JSci Tec 13(2): 197-202.

Bharti and Sharma PC (2014). Prevalence of Extended spectrum beta lactamase (ESBL) producing Pseudomonas aeruginosa strains recovered from Human patients in Himachal Pradesh. Ind J Basic App Med Res 4(1): 430-440.

Biradar A, Farooqui F, Prakash R, Khaqri SY and Itagi I (2016). Aerobic bacteriological profile with antibiogram of pus isolates. Indian J Microbiol Res 3(3): 245-249.

CLSI (2014). Performance Standards for Antimicrobial Susceptibility Testing, Twenty-fourth Informational Supplement, CLSI Document M100-S24, Wayne, PA: Clinical and Laboratory Standards Institute

Dash M, Padhi S, Narasimham MV and Pattnaik $S$ (2014). Antimicrobial resistance pattern of Pseudomonas aeruginosa isolated from various clinical samples in a tertiary care hospital, South Odisha, India. Saudi J Health Sci 3(1): 15-19.

Hassuna NA, Mohamed AHI, Abo- Eleuoon SM and Rizk HAWA (2015). High Prevalence of Multidrug Resistant Pseudomonas aeruginosa Recovered from Infected Burn Wounds in Children. American society Microbiol 6(4): 7.
Hirsch EB and Tam VH (2010). Impact of multidrugresistant Pseudomonas aeruginosa infection on patient outcomes. Expert Rev Pharmacoecon Outcomes Res. 10(4): 441-451.

Khanal S, Joshi DR, Bhatta DR, Devkota U and Pokhrel BM (2013). $\beta$-Lactamase producing multidrugresistant bacterial pathogens from tracheal aspirates of intensive care unit patients at national institute of neurological and allied sciences, Nepal. Int Scholarly Res Notice Microbiol.

Kumar R, Srivastva P, Rishi S, Dahiya SS, Hemwani K and NirwanPS(2012). Detectionand Antimicrobial Susceptibility Pattern of Pseudomonas aeruginosa isolates in various clinical samples with special reference to Metallo beta lactamase from a tertiary care hospital in Jaipur, India. Natl J Med Res 4(2): 128-131.

Forbes BA, Sahm DF and Weissfeld AS (2007). Bailey and Scott's Diagnostic Microbiology 12th edition. Mosby Elsevier Publication, USA: 350-362.

Lee K, Lim YS, Yong D, Yum JH and Chong Y (2003). Evaluation of the Hodge test and the imipenemEDTA double-disk synergy test for differentiating metallo-beta-lactamase-producing isolates of Pseudomonas spp and Acinetobacter spp. J Clin Microbiol. 41: 4623-4632.

Mantravadi HB, Chinthaparthi MR and Shravani V (2015). Aerobic isolates in pus and their antibiotic sensitivity pattern: a study conducted in a teaching hospital in Andhra Pradesh. Int J Med Sci Public Health 4(8): 1076-1079.

Mishra SK, Acharya J, Kattel HP, Koirala J, Rijal BP, Pokhrel BM (2012). Metallo-beta-lactamase Producing Gram-negative Bacterial isolates. J Nepal Health Res Counc 10(22):208-213

Parajuli P, Basnyat SR, Shrestha R, Shah PK and Gurung P (2014). Identification and Antibiotic Susceptibility Pattern of Aerobic Bacterial Wound Isolates In Scheer Memorial Hospital. JSM Microbiol 2(2): 1011.

Patel H and Garala RN (2014). Antibiotic susceptibility pattern of Pseudomonas aeruginosa isolated at SSG hospital Baroda. J Res Med Den Sci2(1): 84-87.

Pathak J and Pokharel N (2015). Multidrug Resistant and Extended Spectrum betalactamase (ESBL) 
Isolates from different clinical specimens. Int $\mathrm{JS} \mathrm{ci}$ Res Pub 5(9): 5.

Poudyal S, Bhatta DR, Shakya G, Upadhyaya B, Dumre SP, Buda G and Kandel BP (2011). ESBL producing multi drug resistant clinical bacterial isolates at National Public Health Laboratory, Nepal. Nepal Med Coll J 13(1): 34-38.

Sharma J, Singh S, Gill AK and Kaur A (2016). Prevalence and Antimicrobial Susceptibility Pattern of Pseudomonas aeruginosa Isolated from Pus Samples in a Tertiary Care Hospital, Bathinda. Int JCon Med Res3 (12): 3481-3483.

Shrestha S, Chaudhari R, Karmacharya SKattel HP, Mishra SK, Dahal RK, Bam N, Banjade N, Rijal BP, Sherchand JB Ohara H, Koirala J and Pokhrel BM (2011). Prevalence ofnosocomial lower respiratory tract infections caused by multidrugresistance pathologens. JInt Microbiol 33(2): 1-17.

Solomon FB, Wadilo FW, Arota AA, Abraham YL (2017). Ann Clin Microbiol Antimicrob 16: 29
Sonth SB, Bhurle A, Gokale S, Hadapad D and Solabannavar SS (2015). Resistance Pattern of Pseudomonas aeruginosa in a Tertiary Care Hospital. Int J Curr Microbiol App Sci 4(10): 919-923.

Soumya S and Nagmoti Mahantesh B (2017). Coexistence of Beta Lactamases (ESBL and MBL) in Pseudomonas aeruginosa Isolates from Pus Samples. Int J Microbiol Res 9(12): pp 981-983.

Tankhiwale S (2016). Beta-lactamases in P. aeruginosa: A threat to clinical therapeutics. Curr Pediatr Res 20(1\&2): 253-257.

Tsering CD, Shyamasree D, Luna A, Ranabir P and Takhellamban SK (2009). Extended spectrum beta-lactamase detection in gram-negative bacilli of nosocomial origin. J Global Infect Dis 1(2): 8792.

Yakha JK, Sharma AT, Dahal N, Lekhak B and Banjara MR (2014). Antibiotic Susceptibility Pattern of Bacterial Isolates Causing Wound Infection Among the Patients Visiting B \& B Hospital. Nepal J Sci Tech 15(2): 91-96. 